\title{
A Novel Robust Controller with Command Filter for Uncertain Morphing Aircraft
}

\author{
Li Wang1,2*, Chunsheng Liư ${ }^{1}$, Huajun Gong1 \\ ${ }^{1}$ Automation Department, Nanjing University of Aeronautics and Astronautics, Nanjing, 210016 China. \\ ${ }^{2}$ Nan Hang Jin Cheng College, Nanjing, 21156 China. \\ * Corresponding author. Tel.: 15301596151; email: li-wang1116@163.com \\ Manuscript submitted July 5, 2017; accepted September 18, 2017. \\ doi: 10.17706/ijcee.2018.10.1.1-11
}

\begin{abstract}
A novel nonlinear NN-based approximation robust control scheme is proposed to solve tracking problem of a class of morphing vehicle in presence of unknown uncertainties and external disturbances. By employing NN to attenuate the system unknown uncertainties, an adaptive feedback control law is designed to automatic disturbance rejection in real time. The NN function reconstruction error is eliminated by the sliding-mode control. The proposed controller can cut down drastically the number of updated parameters, which results in much simpler control algorithm and convenient to implement in applications. Using a signal processing filter into the reference model, robust control is developed to guarantee that the tracked trajectories of system states are very well and smooth without drastically shock. The stability analysis shows that uniformly ultimately bounded of the closed-loop system can be guaranteed. Simulation results demonstrate the effectiveness of the approach.
\end{abstract}

Key words: Robust control, neural network, morphing aircraft, command filter.

\section{Introduction}

Morphing aircraft is the kind of aircraft which can change its aerodynamic configuration in large scale to realize the multi-mission flight and improve its performances. A lot of researches in morphing aircraft have been carried out by countries all over the world [1]-[4], such as the active aeroelastic aircraft structures(3AS) and the morphing aircraft structures(MAS) program and so on. Linear feedback control scheme was developed for adaptive actuator failure compensation of the known linear model, as in [5]. The adaptive feedback control had been used for nonlinear systems with linearly parameterized uncertainty [6]. Automation control methods can provide a satisfactory performance under nominal operating conditions but they can't cope with the environment disturbances during the flight process [7]. Intelligent control were extended to deal with parameter nonlinear uncertainties effectively [8]-[14]. In [11]-[14], adaptive neural network controlling approaches were proposed for nonlinear system to improve control performance. By prior selection of the number of the hidden neurons and prior training, a neural network with a fixed architecture has been used to reduce the system's model errors [12]. However, with the constraints of time and cost, it is hard to get enough sampling data to train a neural network to improve control performance. An online learning direct adaptive RFBNN algorithm was applied to estimate environment disturbances which considered the elements of the weighting vector as estimated parameters [14]. However, with an increase of neural network nodes to improve approximation accuracy, the number of parameter to be estimated will increase significantly. As a result, the online learning time will become prohibitively large. 
Inspired by [15], Bao B M [16] developed a direct NN control method with only one estimated parameter which is the maximum norm of the weighting vector for the strict-feedback nonlinear system. How to deal with the trade-off between online learning time and approximation accuracy for a class of morphing aircraft?

Motivated by the aforementioned work, in this paper, a novel robust adaptive neural track controller is developed for a class of aircraft system subject to time-varying uncertainty and disturbances.

The main contributions of this paper include the following points:

To the best of authors' knowledge, for MIMO nonlinear system, by using NN approximate methods, it is the first time that, the estimated parameter is the norm of the NN weight vector not the weight matrix itself, unlike [15] using only one estimated parameter which is the maximum norm of the weighting vector for SISO nonlinear system. It deals with the trade-off between online learning time and approximation accuracy.

The command filter technique is combined in the novel robust control method. The filters are design to avoid the long shock adjustment time at initial phase which is even harmful to equipment in real systems and guarantee the system tracking performance.

The rest of this paper is organized as follows. In Section 2, we present the statement of the ICE morphing aircraft and the nominal system for uncertain. In Section 3, we design an online NN-based adaptive control scheme to guarantee the system tracking performance. Finally, the concluding remarks are addressed in Section 4.

Notations: $R_{\mathrm{m}}$ denotes the Euclidean space of all real m-vectors. $R^{n \times m}$ denotes the space of all real matrices. $W_{*_{i}}$ denotes the $i^{\text {th }}$ column vector of the matrix $W$.

\section{Description of ICE Morphing Aircraft}

The aircraft model is the Innovative Control Effector (ICE) morphing aircraft whose configuration is a tailless, delta-wing fighter used for the purpose of study novel control effect or concepts [17]. For the ICE morphing aircraft (MA) model configuration, hundreds of distributed shape-change devices arrays are used to compose the effector suite employed to stabilize and maneuver the air vehicle, replacing the conventional hinged surfaces for ailerons. As illustrated by Fig.1, the effector suite includes four arrays with 78 devices on each wing [18]. The 78 devices on each wing are arranged as follows: 10 devices in the upper-surface leading-edge (ULE) array, 22 devices in the lower-surface trailing-edge (LTE) array, 22 devices in the upper-surface trailing-edge (UTE) array, and 24 devices in the upper-surface wingtip (UTip) array.

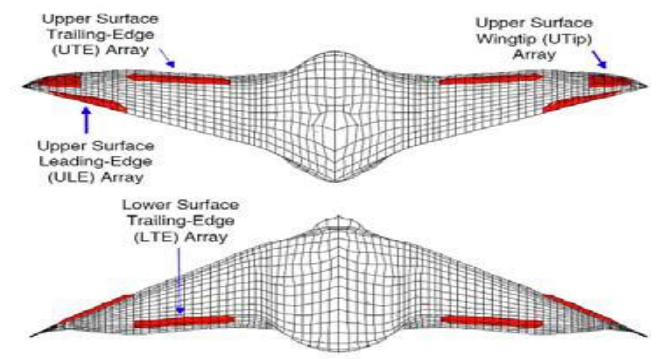

Fig. 1. Shape-change actuator arrays of the ICE aircraft.

The lower-surface trailing-edge, upper-surface trailing-edge, upper-surface and leading-edge arrays all have a maximum displacement height of 2.4 inches. The upper-surface wingtip array has a maximum displacement height of 1.2 inches. The lateral dynamics of a generic ICE aircraft at flight condition of Mach 0.6 and $15000 \mathrm{ft}$ considered in this paper is with the following equations: 


$$
\begin{aligned}
& \dot{v}=w \cdot p-u \cdot r+g\left(C_{y} \cdot \text { qbar } \cdot S / W+\cos (\theta) \sin (\phi)\right) \\
& \dot{p}=\left(I_{z} \cdot L+I_{x z} \cdot N\right) /\left(I_{x} \cdot I_{z}-I_{x z}{ }^{2}\right) \\
& \dot{r}=\left(I_{x z} \cdot L+I_{x} \cdot N\right) /\left(I_{x} \cdot I_{z}-I_{x z}{ }^{2}\right) \\
& \dot{\phi}=r
\end{aligned}
$$

where $g$ is gravity. The $(u, v, w)$ and $(p, q, r)$ are the translation and rotation rates about the body-fixed, reference $(x, y, z)$ axes of the aircraft, respectively.

The model is comprised four state variables, $x=\left[\begin{array}{llll}1 & p & r & \phi\end{array}\right]^{T}, \quad v, p, r, \phi$ denote the body-axis lateral velocity $(\mathrm{ft} / \mathrm{s})$, roll rate $(\mathrm{deg} / \mathrm{s})$, yaw rate $(\mathrm{deg} / \mathrm{s})$, roll angle respectively. $\mathrm{L}, \mathrm{N}$ represents total rolling and yawing moment respectively. The detail is as follows:

$$
\begin{aligned}
& L=C_{l} \cdot q b a r \cdot S \cdot b+\left(I_{y}-I_{z}\right) \cdot q \cdot r+p \cdot q \cdot I_{x z} \\
& N=C_{n} \cdot q b a r \cdot S \cdot b+\left(I_{x}-I_{y}\right) \cdot q \cdot p+q \cdot r \cdot H_{T}
\end{aligned}
$$

where $I_{x}, I_{y}, I_{z}$ are the moments of inertia about the $x, y, z$ axes and $I_{x z}$ is the relevant product of inertia about the y axis.

Total air speed and dynamic pressure qbar are calculated as:

$$
q b a r=\rho \cdot V^{2} / 2, V^{2}=u^{2}+v^{2}+w^{2}
$$

The detail of the parameter values can be found in [17]. The lateral dynamics of the open-loop aircraft model are represented in state-space form is

$$
\dot{x}=A x(t)+B u(t)
$$

where $x(t)=[v, p, r, \phi]^{T}$ are the state variables. The control input vector $u=\left[\delta_{\text {LTEL }}, \delta_{\text {UTEL }}, \delta_{\text {ULEL }}, \delta_{\text {UTTPR }}\right]^{T}$ is from the four morphing actuator arrays as defined in Fig.1. The matrices $A \in R^{n \times n}$ and $B \in R^{n \times m}$ contain the aircraft stability coefficients and control coefficients.

The MA system has inherently uncertainties, varying dynamics due to the alteration of their configuration, modeling errors and environment disturbances. So the dynamic model of MA system can be described as

$$
\dot{x}=A x+B u(t)+f(x)+D d(t)
$$

where $A, B, D$ are appropriately dimensional known matrices. The $d(t)$ is additional external unknown disturbance which changed at a limited rate, its time derivative is norm bounded i.e. $\|d(t)\|^{2} \leq \alpha,\|\dot{d}(t)\|^{2} \leq \eta$, $(\alpha, \eta>0)$. The $f(x)$ is bounded differential smoothly unknown modeling error caused by the wing shape change due to different flight missions.

For the most of the flight conditions considered, the open-loop dynamic model is unstable and non-minimum phase.

Assumption 1:

1) The matrix pair $(A, B)$ is controllable;

2) The input $u$ lies into a compact set: $\Omega=\left\{u \mid u_{\min } \leq u \leq u_{\max }\right\}, u_{\max }, u_{\min }$ are the upper-lower limit line of the input $u$. 


\section{Novel Controller Design and Stability Analysis}

To deal with the modeling errors and environment disturbances, during the flight processing, a novel adaptive neural-based control strategy is shown in Fig. 2. $x_{\text {ref }}$ are desired reference signals and $x_{\text {vir-ref }}$ are virtual reference signal after signal processing filter to guarantee the system tracking performance.

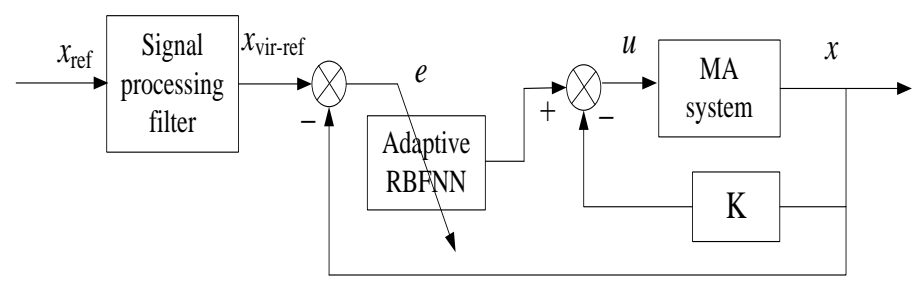

Fig. 2. Control flowchart of the MA system.

In control engineering, RBFNN With a finite number of hidden neurons can approximate a nonlinear function as accurate as possible [19-22]. The continuous function $f(x)$ over the compact set $A_{d} \subset R^{n}$ can be approximated by the RBFNN to any accuracy as

$$
f(x)=B_{0} W^{* T} S(x)+B_{0} \xi(x)
$$

where $W^{*} \in R^{N}$ is ideal constant vector for some integer $\mathrm{N}$ only required for analytic purposes. They $\xi(x)=\left[\xi_{1}, \xi_{2}, \ldots \xi_{k}\right]^{T}$ are the neural network approximation error. $S(x) \in R^{k}$ is the so-called radial basis function of the RBFNN hidden node. $B_{0}$ is the designed matrix to avoid solving pseudo-inverse gain matrix.

Assumption2: $|\xi| \leq \xi_{0}, \xi_{0}$ is the unknown positive constant on the compact set $A_{d}$.

Consequently, equation (5) can be written as

$$
\dot{x}=A x+B u(t)+B_{0} W^{*} S(x)+B_{0} \xi(x)+D d(t)
$$

The objection of the control design is to arrive at a strategy that the states of the plant follow those of a reference model.

In this paper, the controller is developed using parameter adaptation and adaptive RBFNN combined with pole placement control scheme. The main idea is that adaptive RBFNN to attenuate the system unknown uncertainties, adaptive parameter updated law is designed to automatic disturbance rejection in real time.

The conventional adaptive approach is used to estimate the RBFNN weight matrix themselves. We introduce the novel unknown constant $\theta$ which is specified as

$$
\theta=\left[\begin{array}{llll}
\theta_{1} & \theta_{2} & \ldots & \theta_{n}
\end{array}\right]^{T} \theta_{i}=\left\|W_{*_{i}}^{*}\right\|
$$

where $\theta_{i}$ is the norm of the $W_{*_{i}}{ }^{*}$ and an unknown positive constant because of the unknown.

Remark1: If the number of RBFNN layers is 4,15,1 respectively, the novel adaptive RBFNN algorithm includes 4 only update parameters in this paper, the traditional RBFNN algorithm contain 60 parameters correlation with the number of hidden layers nodes. It guaranteed that the computational burden of the algorithm can drastically be reduced and the algorithm is convenient to implement in applications and the estimated accuracy is not affected. In this way, the adaptive RBFNN algorithm contained only n parameters corresponding to the order of the system which reduce computation complexity of the control algorithm. 
The linear time-invariant asymptotically stable reference model is chosen as follows

$$
\dot{x}_{m}(t)=A_{m} x_{m}(t)+B_{m} r(t)
$$

where $x_{m} \in R^{n}, \quad r(t) \in R^{r}, A_{m} \in R^{n \times n}$ is Hurwitz matrix. Generally reference input is chosen as a square wave.

An adaptive flight control law is designed the control input $u(t)$ to assure that the system (7) can track a desired reference model (9) or to eliminate the system uncertainties and external distances effectively.

Based on the pole assignment theory, a suitable matrix $K_{1}, K_{2}$ can be chosen to assure linear system stability as

$$
A_{m}=A-B K_{1}, \quad B_{m}=B K_{2}
$$

The adaptive RBFNN and parameter adaptation is updated online to improve control dynamic performance. The adaptive control input can be constructed as the following form:

$$
u(t)=-K_{1} x(t)+K_{2} r(t)+\hat{v}_{N}+\hat{v}_{b}+v_{s}
$$

where $\hat{v}_{N}$ is the uncertainty estimation generated by the output the adaptive RBFNN, $\hat{v}_{b}$ is the parameter updated law online for disturbance estimation, vs is used to attenuate the approximation error of the RBFNN. The design virtual controllers $\hat{v}_{N}$ and $\hat{v}_{b}$ are derived in the sense of Lyapunov function.

The control law is design as

$$
\begin{gathered}
\hat{v}_{N}=-\frac{1}{2} K_{f} \hat{\theta} S^{T}(x) S(x) \\
\hat{v}_{b}=-K_{d} \hat{d}(t)
\end{gathered}
$$

where $\hat{\theta} \in R^{n}, \hat{d}(t)$ are the estimations of $\theta$ and $\mathrm{d}(\mathrm{t})$, respectively. The uncertainty and disturbance can be approximated and eliminated by using them. The matrices $K_{f}, K_{d}$ are designed to $B K_{f}=B_{0}, B K_{d}=D$.

Lemma1: If the sliding model virtual control law is adopted as

$$
v_{s}=-\xi_{0} K_{f} \operatorname{sgn}\left(Q^{T} e\right)
$$

Then $e^{T} Q \xi+e^{T} Q K_{f}^{-1} v_{s} \leq 0$. where $Q=P B_{0}$.

Proof: from equation (14), we get

$$
e^{T} Q \xi+e^{T} Q K_{f}^{-1} v_{s}=e^{T} Q \xi-\xi_{0} e^{T} Q \operatorname{sgn}\left(Q^{T} e\right) \leq\left\|\xi^{T} Q e\right\|-\xi_{0} e^{T} Q \operatorname{sgn}\left(Q^{T} e\right) \leq\left(\left\|\xi^{T}\right\|-\xi_{0}\right) e^{T} Q \operatorname{sgn}\left(Q^{T} e\right) \leq 0
$$

Based on (10) and the control laws (11), (12), (13) the closed-loop control system is rewritten as

$$
\dot{x}(t)=A_{m} x(t)+B_{m} r(t)+B_{0} W^{* T} S(x)+B_{0} \xi(x)+B v_{s}+D d(t)-\frac{1}{2} B_{0} \hat{\theta} S^{T}(x) S(x)-\mathrm{D} \hat{d}(t)
$$

Defining the tracking error $e(t)=x(t)-x_{m}(t)$, the error dynamic model can be defined 


$$
\dot{e}(t)=A_{m} e(t)+B_{0} W^{* T} S(x)+B_{0} \xi(x)+B v_{s}+D \tilde{d}(t)-\frac{1}{2} B_{0} \hat{\theta} S^{T}(x) S(x)
$$

where $\tilde{d}(t)=d(t)-\hat{d}(t)$.

Lemma2: For $\forall x \in R^{n}$

$$
\begin{gathered}
W_{*_{i}}{ }^{* T} S(x) \leq \frac{1}{2} W_{*_{i}}{ }^{*} W_{*_{i}}{ }^{* T} S(x)^{T} S(x)+\frac{1}{2} \leq \frac{1}{2} \theta_{i} S(x)^{T} S(x)+\frac{1}{2} \\
W^{* T} S(x) \leq \frac{1}{2} \theta S(x)^{T} S(x)+\frac{1}{2} I_{\mathrm{n} \times 1}
\end{gathered}
$$

where $\theta_{i}, \theta$, are defined as in (8). $I_{\mathrm{n} \times 1}=[1, \ldots, 1]^{T}{ }_{n \times 1}$ is $n \times 1$ identity column vector.

Lemma3: If the adaptive parameters update law is designed

$$
\dot{\hat{d}}^{T}=\left\{\begin{array}{cl}
\psi, & \text { otherwise } \\
\psi+\left(\|\hat{d}\|^{2}-\alpha\right) \operatorname{tr}(\hat{d} \psi) \hat{d}^{T} & \text { if }\|\hat{d}\|^{2}>\alpha \text { and } \operatorname{tr}(\hat{d} \psi)<0
\end{array}\right.
$$

Then $\left(\dot{\hat{d}}^{T}-\psi\right) M \tilde{d} \leq 0$. where $\psi=e^{T} P D M^{-1}, \quad M^{T}=M>0, \quad P^{T}=P>0$.

Proof is similar to the one in the reference [23].

Remark 2: From Lemma3, the parameters do not drift away and stay with known bounded region even in the presence of external disturbance. From $\|d(t)\|^{2} \leq \alpha$ and parameter updating law (18), $\|\tilde{d}(t)\|$ is also bounded, i.e. $\|\tilde{d}(t)\|^{2} \leq \eta_{0}$.

In order to guarantee certain desired trajectory properties of system (5), the following RBFNN parameter update law is designed

$$
\dot{\tilde{\theta}}=-\left[k_{\theta} e Q S(e)^{T} S(e)\right] / 2
$$

where $k_{\theta}$ is a positive constant, $\tilde{\theta}=\theta-\hat{\theta}, \dot{\tilde{\theta}}=-\dot{\hat{\theta}}$.

Theorem1: Consider adaptive flight control law (11), (12), (13), (14), the parameter adaptation update law (20) and (21), the uncertainty and disturbance can be eliminated and state error $e(t)$ is uniformly ultimately bounded, that is $e \in L_{\infty}, \lim _{t \rightarrow \infty}\left|x-x_{m}\right|$.

Proof: Consider the candidate Lyapunov function as

$$
V=\frac{1}{2} e^{T} P e+\frac{1}{2 k_{\theta}} \tilde{\theta}^{T} \theta+\frac{1}{2} \tilde{d}^{T} M \tilde{d}
$$

where $P$ is the symmetric definite matrix solution of $-\left(A_{m}{ }^{T} P+P A_{m}\right)=G>0, G$ is symmetric definite matrix.

The time derivative of $V(t)$ is obtained

$$
\dot{V}=-\frac{1}{2} e^{T} G e+e^{T} Q W^{* T} S(e)-e^{T} P D \tilde{d}+\frac{1}{k_{\theta}} \tilde{\theta}^{T} \dot{\tilde{\theta}}+\dot{\tilde{d}}^{T} M \tilde{d}+e^{T} Q \xi+e^{T} Q K_{f}^{-1} v_{s}
$$

From lemma 1, we obtain 


$$
\dot{V} \leq-\frac{1}{2} e^{T} G e+e^{T} Q W *^{T} S(e)-e^{T} P D \tilde{d}+\frac{1}{k_{\theta}} \tilde{\theta}^{T} \dot{\tilde{\theta}}+\dot{\tilde{d}}^{T} M \tilde{d}
$$

From lemma 3, we get

$$
-e^{T} P D \tilde{d}+\dot{\tilde{d}}^{T} M \tilde{d} \leq\left(\dot{\hat{d}}^{T}-\psi\right) M \tilde{d}-\dot{d}^{T} M \tilde{d} \leq-\dot{d}^{T} M \tilde{d} \leq-\lambda_{\min }(M)\left(\eta_{0}+\eta\right) \leq 0
$$

By applying (21), (25) and lemma2, (24) can be rewritten as

$$
\dot{V} \leq-\frac{1}{2} e^{T} G e+\frac{1}{2} e^{T} Q \leq-\frac{1}{2} \lambda_{\min }(G)\|e\|^{2}+\frac{1}{2}\|e\| .\|Q\|
$$

where $\lambda_{\max }(G)$ is the minimum eigenvalue of G. Choosing the suitable design parameters $\lambda_{\text {min }}(G) \geq\|Q\|$, thus $\dot{V} \leq 0$, which imply $e \in L_{\infty}$. The error dynamic model of the system is asymptotically stable. Then, it can be proved that the tracking error $e \rightarrow 0$ as $t \rightarrow \infty$. That is to say, asymptotic tracking is achieved.

The goal in this study is to design a dynamic input $u$ to steer the unstable system from a given set of system states $x$ to desired states xm. At initial phase, the big difference between $x$ and xm may result in taking a long shock adjustment time, even harmful to equipment in real systems. Some filters are design to avoid the harmfulness and guarantee the system tracking performance.

The filter is design as follow:

$$
E(t) \dot{x}_{r}+x_{r}=x_{m}
$$

where $x m$ and $x_{\mathrm{r}}$ are the input and output of the filter, respectively.

The goal of the signal processing filter is to obtain virtual reference signals xr which track the system desired reference signals $\mathrm{xm}$. The initial condition of the filter is design as $x_{\mathrm{r}}(0)=x(0)$ to guarantee all the error terms are very small at the beginning of the actual control. The filter parameter is chosen as

$$
E(t)=E_{0} e^{-\omega t}+\delta, \omega>0, \delta>0
$$

If the parameter $\delta$ is sufficiently small, the ultimate difference between the actual reference signal $x_{\mathrm{m}}$ and the virtual reference signal $x_{\mathrm{r}}$ is also sufficiently small. Thus as long as the system output is capable to track the virtual reference signal very well, good actual tracking performance can be obtained accordingly.

\section{Simulation}

In this section, an ICE morphing aircraft model [17] (at flight condition of Mach 0.6 and 15000ft) will be used to verify the effectiveness of the proposed controller and the state and input matrices $(A, B, D)$ are given as following

$$
A=\left[\begin{array}{cccc}
-0.0134 & 48.5474 & -632.3724 & 32.0756 \\
-0.0199 & -0.1209 & 0.1628 & 0 \\
-0.0024 & -0.0526 & -0.0252 & 0 \\
0 & 1 & 0.0768 & 0
\end{array}\right], B=\left[\begin{array}{cccc}
0 & 0 & 0 & 0 \\
-0.0431 & 0.0476 & -0.0401 & -0.0308 \\
-0.0076 & -0.0023 & -0.0022 & 0.0297 \\
0 & 0 & 0 & 0
\end{array}\right] \quad D=\left[\begin{array}{ccc}
0 & 0 & 0 \\
10.00 & 0 & 15.00 \\
6.00 & 3.00 & -0.00 \\
0 & 0 & 0
\end{array}\right] .
$$

The open-loop poles of the aforementioned system are: -1.2795 for the roll mode, -0.0227 for the spiral mode, and $0.5714 \pm 0.7711 i$ for the Dutch-roll mode. The open-loop ICE model has unstable Dutch-roll mode. 
The desired reference state vector $x_{\mathrm{m}}(t)$ is generated with [5]

$$
A_{m}=\left[\begin{array}{cccc}
-0.0134 & 48.5474 & -632.3724 & 32.0756 \\
0.5386 & -1.7746 & -23.8313 & -4.8526 \\
0.0664 & 0.6431 & -11.2476 & -0.1192 \\
0 & 1.0000 & 0.0768 & 0
\end{array}\right], \quad B_{m}=[0,1,0,0]
$$

The reference input signal $r(t)$ is chosen to generate a doublet roll angle and square waves.

$$
r(t)=\left\{\begin{array}{cc}
0.5 & 10 i \leq t \leq 0 i+5, i=0,1,2, \ldots \\
-0.5 & \text { otherwise }
\end{array}\right.
$$

The uncertainty vector is $f(x)=\left[1.0+0.5 x 3 \sin \left(x_{1}\right), \quad-0.133+0.35 \cos \left(x_{3}\right), \quad-0.230+0.223 \cos \left(x_{2}\right)\right.$, $\left.-0.7+0.9 \sin \left(x_{4}\right)\right] \mathrm{T}$, the external disturbance vector is $d(t)=[0.01 \sin (0.5 t), 0.02+0.05 \cos (0.5 t), 0.1 \cos (0.5 t)]^{\mathrm{T}}$, the initial states are chosen as $x_{\mathrm{m}}(0)=[0,0,0,0]^{\mathrm{T}}$ and $x_{\mathrm{r}}(0)=x(0)=[0.75,0.5,0.052,0.06]^{\mathrm{T}}$. The parameters used for reference signal processing are $E_{0}=1$, The designed matrix parameters are

$$
\begin{gathered}
K_{1}=\left[\begin{array}{cccc}
5.2024 & -3.6518 & -327.5860 & -35.4384 \\
-4.2705 & 10.5167 & 201.4834 & 35.2920 \\
4.1684 & -6.3510 & -231.7635 & -31.2196 \\
-1.1284 & -23.9103 & 292.5481 & -12.6883
\end{array}\right], K_{2}=[-7.6257,7.3330,-6.5989,-1.8723]^{T}, \\
K_{f}=\left[\begin{array}{cccc}
0 & -7.6257 & -12.8844 & 0 \\
0 & 7.3330 & 2.2757 & 0 \\
0 & -6.5989 & 2.2757 & 0 \\
0 & -1.8723 & 30.0646 & 0
\end{array}\right], \quad K_{d}=\left[\begin{array}{cccc}
-153.5631 & -38.6532 & -114.3851 \\
86.9841 & 6.8270 & 109.9952 \\
-105.2435 & -19.6273 & -98.9835 \\
161.6649 & 90.1939 & -28.0842
\end{array}\right]
\end{gathered}
$$

Fig. 3, Fig.4, Fig. 5, and Fig. 6 are obtained simulation results in this study. As depicted in Fig. 3, the virtual signal xr can track the actually reference states signal xm very well only after about two seconds. From Fig. 4, without processing signal filter, between system states $x=\left[x_{1}, x_{2}, x_{3}, x_{4}\right]$ and reference states $x \mathrm{~m}=\left[\mathrm{xm}_{1}\right.$, $\left.\left.x m_{2}\right), x m_{3}, x m_{4}\right]$, there exist the very poor tracking dynamic performance because of large overshoot and drastically shock adjustment at initial phase which harmful to equipment in real systems. With the command filter design, the system state $\mathrm{x}$ could track the new virtual reference signals $x_{\mathrm{r}}$ very well without drastically shock presented in Fig. 5. The Fig. 6 gives the control signals of the closed-loop system.
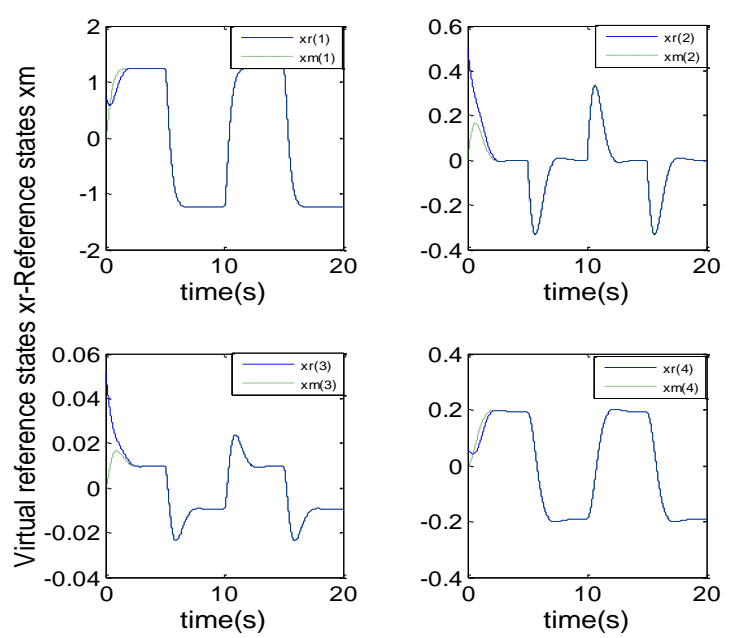

Fig. 3. The duration curves of visual reference states $x_{\mathrm{r}}$ and actual reference states $x_{\mathrm{m}}$. 

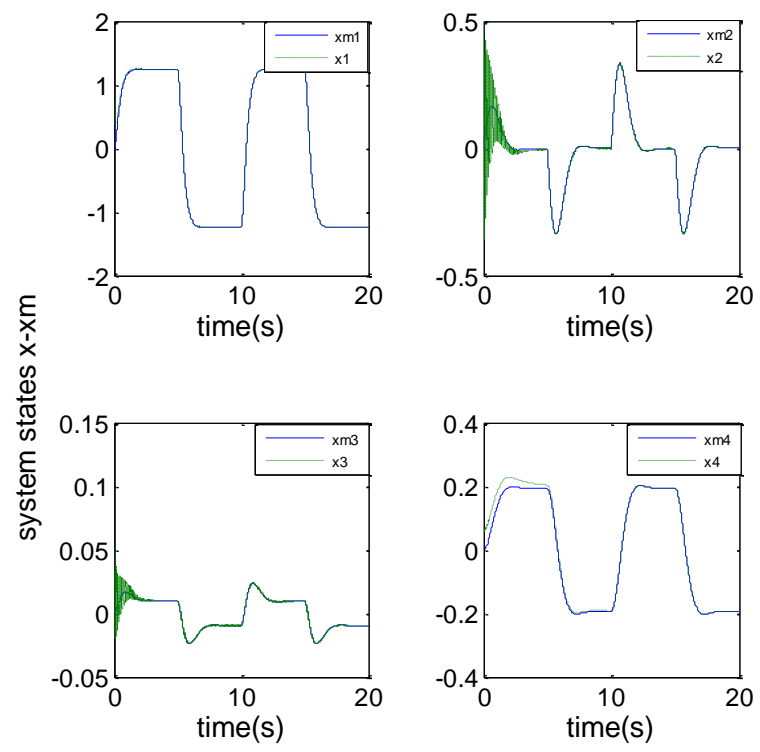

Fig. 4. The states tracking $x-x_{\mathrm{m}}$ of the system.

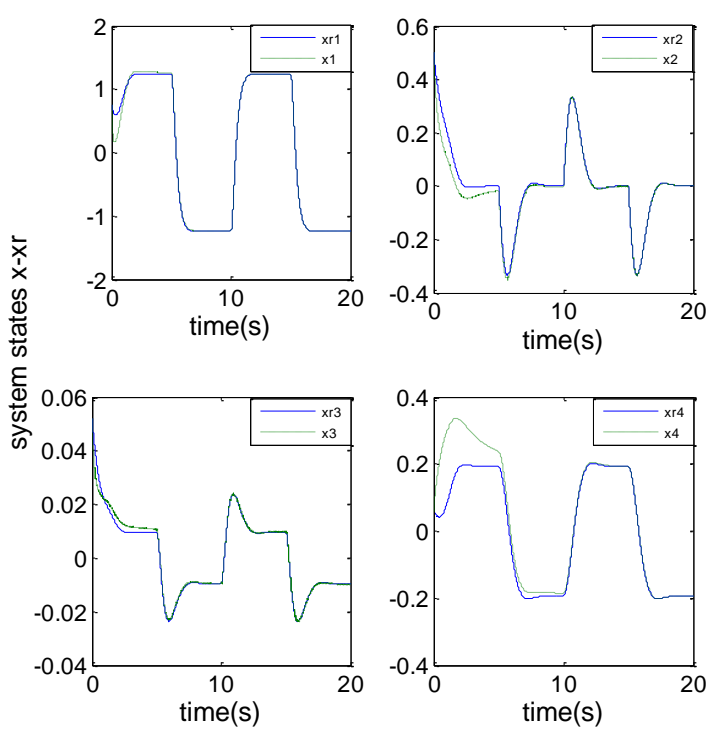

Fig. 5. The state tracking $x-x r$ of the system.

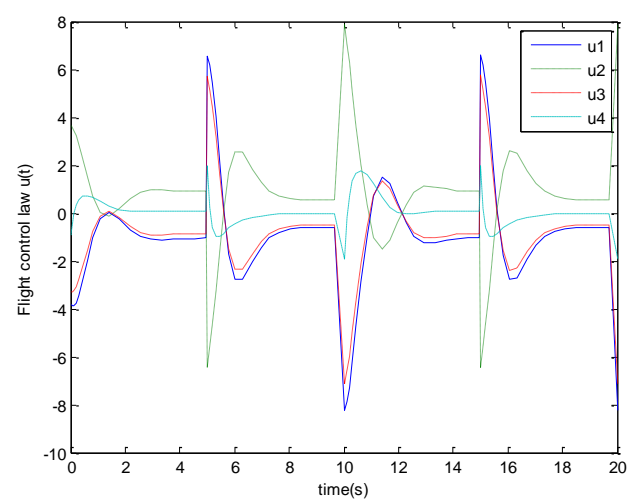

Fig. 6. Control input $u(t)$. 


\section{Conclusion}

In this paper, combined the signal filter with adaptive pole assignment approach which is a nonlinear NN-based robust control scheme is proposed for a genetic morphing aircraft subject to uncertainties and disturbances. The feature of the designed control algorithm is simplicity. The number of adaptive updated parameter of RBFNN is only same as order of the system, no matter how many neural networks nodes are used. This reduces the computation load of the control algorithm and estimated accuracy is not affected. By adding a signal processing filter into the reference model, the smooth tracked trajectories of system states are guaranteed. The proof of convergence for the robust NN-based control is presented. From theory analysis and simulation results, the stability and tracking performance of the morphing aircraft with uncertainties is guaranteed effectively. Our future research will concentrate on devising novel parameters updating laws and control scheme rendering the asymptotically stability of all the signals of the closed-loop system based-on the results of this paper.

\section{Acknowledgment}

This work was supported by National Natural Science Foundation of China under Grant 61074063.

\section{References}

[1] Schweiger, J., Suleman, A., Kuzmina, S., \& Chedrik, V. (2002). MDO concepts for an European research project on active aeroelastic aircraft. AIAA-0240871.

[2] Bowman, J., Sanders, B., \& Cannon, B. (2007). Development of next generation morphing aircraft structures. AIAA-2007-1730.

[3] Pendleton, E., \& Griffin, K. E. (1996). A flight research program for active aeroelastic wing technology. AIAA-96-27023.

[4] Boyd, P. I., Cole, S., \& Miller, G. (1992). A summary of the active flexible wing program. Dynamics Specialists Conference, 32.

[5] Tao, G., Chen, S., Fei, J., \& Joshi, S. M. (2004). An adaptive actuator failure compensation scheme for controlling a morphing aircraft model. Proceedings of The 42nd IEEE Conference on decision and Control, Vol. 5 (pp. 4926-4931).

[6] Kenneth, B., Kristin, F., \& Rick, L. (2005). Controllers for disturbance rejection for a linear Input-Varying class of morphing aircraft. Proceedings of the 46th AIAA Conference on Structures, Structural Dynamics \& Materials (pp. 1-14).

[7] Carlo, L. B., Fabio, L., \& Giorgio, M. (2012). Efficient rotorcraft trajectory optimization using comprehensive models by improved shooting methods. Aerospace science and technology, 23(1), 34-42.

[8] Gandhi, N., Jha, A., \& Monaco, J. (2007). Intelligent control of a morphing aircraft. Proceedings of the 46th AIAA Conference on Structures, Structural Dynamics \& Materials (pp. 1-17).

[9] Liu, C., Chen, W., \& John, A. (2012). Tracking control of small scale helicopters using explicit nonlinear MPC augmented with disturbance observers. Control Engineering Practice, 20(3), 258-268.

[10] Sun, L. Y., \& Liu, Y. (2010). Adaptive backstepping control of nonlinearly parameterized systems with time-varying disturbances. Proceedings of the 29th Chinese Control Conference (pp. 2207-2211).

[11] Ge, S. S., Hang, C. C., \& Tao, Z. (1999). Adaptive neural network control of nonlinear systems by state and output feedback. IEEE Transactions on Systems Man and Cybernetics, 29(6), 818-828.

[12] Vargas, J. A. R., \& Hemerly, E. M. (1999). Robust Neural Adaptive Observer for MIMO Nonlinear Systems. Proceedings of IEEE SMC '99 Conference on System, Man and Cybemetics (pp. 1084-1089).

[13] Ruiz, V. J., \& Hemerly, E. M. (2000). Neural adaptive observer for general nonlinear systems. Proceedings of American Control Conference (pp.708-712). 
[14] Hao, C., Yu, G., \& Xia, H. (2013). Online modeling with tunable RBF network. IEEE Transactions on Cybernetics, 43(3), 935-9472.

[15] Wang, H. Q., Chen, B., \& Lin, C. (2012). Direct adaptive neural control for strict-feedback stochastic nonlinear systems. Nonlinear Dynamics, 67(2), 2703-2718.

[16] Bao, B. M., \& Ties, H. L. (2015). A novel neural network-based adaptive control for a class of uncertain nonlinear systems in strict-feedback form. Nonlinear Dynamics, 79(2), 1005-1013.

[17] Raney, D. L., Montgomery, R. C., \& Green, L. L. (2000). Flight control using distributed shape-change effector arrays. Proceedings of the 41st AIAA Conference on Structures, Structural Dynamics and Materials (pp. 1-12).

[18] Liu, C. S., Li, C. T., \& He, Z. (2012). Adaptive control of a morphing aircraft through updating the number of actuators. Proceedings of AIAA 2012-4446 Guidance, Navigation and Control Conference (pp.1-9).

[19] Vargas, J. A. R., \& Hemery, E. M. (2000). Robust neural adaptive observer for general nonlinear systems. Proceedings of American control conference (pp.708-712).

[20] Schilling, J. J., Carroll, J. A., \& Ajlouni, F. (2001). Approximation of nonlinear systems with radial basis function neural networks. IEEE Trans on Neural Network, 12(1), 1-15.

[21] Yao, W., Chen, X. Q., \& Luo, W. C. (2009). A gradient-based sequential radial basis function neural network modeling method. Neural Computing and Applications, 18(5), 474-484.

[22] Liu, C. S., Zhang, S. J., \& Hu, S. S. (2008). Adaptive neural-network-based fault detection and diagnosis using unmeasured states. International Journal on IET Control Theory Applications, 2(12),1066-1076.

[23] Liu, C. S., \& Zhang, S. J. (2013). A novel robust control framework applied to a morphing aircraft. Journal of Systems of Engineering and Electronics, 24(2), 281-287.

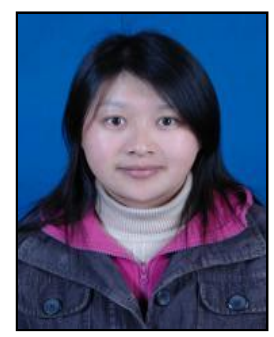

Li Wang received his M.E. degree from Nanjing University of Aeronautics and Astronautics (NUAA). She is now a lecturer in NanHang Jin Cheng College and working toward the Ph.D. degree in automation engineering in NUAA. Her current research interests include optimal control, adaptive control and intelligent control with the application.

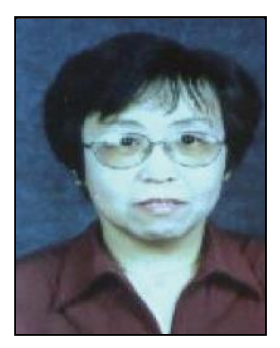

Chunsheng Liu received her B.S., M.E. and Ph.D. degrees from Huazhong University of Science \& Technology, Xi'an Jiaotong University and Nanjing University of Aeronautics and Astronautics (NUAA), respectively. She is now a professor and Ph.D. supervisor in automation engineering in NUAA. Her current research interests include adaptive control, fault diagnosis and tolerant control with the application aircraft.

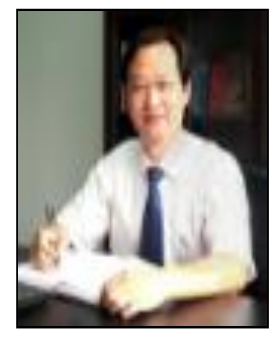

Huajun Gong received his Ph.D. degree from Nanjing University of Aeronautics and Astronautics (NUAA). He is now a professor and Ph.D. supervisor in automation engineering in NUAA. His current research interests include flight integrated control, system modeling and simulation, fly-by-light (FBL) control system and the application. 